\title{
Multimodality Approach to Characterizing the Distinctive Hallmarks of Lung Fibrosis: A Mouse Model with Bleomycin and Indocyanine Green
}

\section{Andrea Grandi}

Chiesi Farmaceutici Spa https://orcid.org/0000-0001-7625-1381

\section{Erica Ferrini}

University of Parma: Universita degli Studi di Parma

\section{Roberta Ciccimarra}

University of Parma: Universita degli Studi di Parma

Martina Mambrini

University of Parma: Universita degli Studi di Parma

\section{Laura Mecozzi}

University of Parma: Universita degli Studi di Parma

\section{Ludovica Leo}

University of Parma: Universita degli Studi di Parma

\section{Mario Silva}

University of Parma: Universita degli Studi di Parma

Nicola Sverzellati

University of Parma: Universita degli Studi di Parma

\section{Gaetano Donofrio}

University of Parma: Universita degli Studi di Parma

\section{Livia Ruffini}

University of Parma: Universita degli Studi di Parma

\section{Gino Villetti}

Chiesi Farmaceutici Spa

Franco Fabio Stellari ( $\nabla$ fb.stellari@chiesi.com )

Chiesi Farmaceutici Spa

\section{Research}

Keywords: Indocyanine Green, Bleomycin, Pulmonary fibrosis, Emphysema-like, Animal model, Fluorescent imaging, Micro-CT

Posted Date: June 29th, 2021 
DOl: https://doi.org/10.21203/rs.3.rs-615697/v1

License: (c) (1) This work is licensed under a Creative Commons Attribution 4.0 International License. Read Full License 


\section{Abstract}

\section{Background.}

Idiopathic Pulmonary Fibrosis is a progressive disease with short life expectancy and no diseasemodifying pharmacological therapy. The continuous refinement of animal models and the integration of in-vivo imaging techniques is fundamental for the selection of new antifibrotic drugs.

Indocyanine Green (ICG), a fluorescent dye, was administered by oropharyngeal aspiration (OA) to mice with Bleomycin (BLM) to map the lung exposure.

\section{Methods.}

Female mice C57bl/6 were treated via OA with BLM+ICG or ICG. Animals were imaged at 7, 14 and 21 days either with the fluorescent system or Micro-CT. At each time point subsets of mice were sampled for ex-vivo assessment.

Histological assessment of fibrosis by Ashcroft score, airspace enlargements and mean linear intercept (MLI) were evaluated at 7, 14 and 21 days. Leukocytes and cytokines were measured in bronchoalveolar lavage fluid.

\section{Results.}

Fluorescence imaging revealed a persistent lung signal in both groups until 21 days. In BLM+ICG group, Micro-CT detected a marked increase in hypo- and non-aerated tissues throughout the study. At later time points hyper-inflated tissue was detected. Histology revealed high Ashcroft score throughout the timecourse with a prominent increase in airspace size and MLI at day 21. ICG mice had healthy lungs.

\section{Conclusions.}

We showed that ICG can be used as a tracer to map the distribution of BLM in lungs. However, BLM+ICG produced unexpected severe lung changes different from pure BLM model, such as emphysema-like features which progressively worsened. The multimodalities approach warranted characterization of the distinctive features of this new pulmonary fibrosis model and provided fundamentals for in-vivo translation.

\section{Background}

Idiopathic Pulmonary Fibrosis (IPF) is a heterogenous, chronic disease characterized by progressive lung scarring and impaired pulmonary function, ultimately leading to respiratory failure and death. A resolutive pharmacological therapy has yet to be identified, with lung transplantation often remaining the only treatment option [1, 2]. Even though animal models of IPF are crucial to satisfy this urgent unmet medical need, none of the currently available models can fully replicate the human disease [3, 4]. Among the 
animal models of IPF, administration of the chemotherapeutic agent bleomycin (BLM) in mice is the most widely used [5]. However, this model presents some drawbacks, which are challenging to predict and standardize, such as spontaneous resolution of fibrosis, variability and mortality. Nonetheless, preclinical data are needed for selection of the best drug candidate for clinical trial and then clinical use, thus the continuous refinement of animal models is fundamental, as is the integration of in-vivo imaging techniques in preclinical studies [6].

Imaging technologies play an important role in decreasing intra-experimental variability, and, as a result, significantly reduce the number of animals used per experiment fully in compliance with the 3R's principles [13]. Notably, thoracic imaging represents a surrogate outcome in clinical trials and clinical practice; therefore, the development of preclinical imaging model is expected to integrate well with optimized drug development.

In the present work, Indocyanine Green (ICG), a water-soluble fluorescent dye, was mixed with BLM and the solution was administered by oropharyngeal aspiration (OA) to mice.

ICG has been used as tracer to elucidate the distribution and exposure of BLM in the lungs, in light of its common use for clinical and preclinical applications [7-9].

We sought to analyze a new model of lung fibrosis in mice based on a single OA and with multimodality approach for characterization of the disease progression.

\section{Materials And Methods}

\section{Experimental Animals}

Female inbred C57BI/6j (8-weeks old, $20 \mathrm{~g}$ of body weight) mice were purchased from Envigo, Italy (San Pietro al Natisone, Udine, Italy). Animals were housed five per cage at Chiesi Farmaceutici animal facility. Prior to use, animals were acclimatized for at least 7 days to the local vivarium conditions (room temperature: $20-24^{\circ} \mathrm{C}$; relative humidity: $40-70 \%$; 12 -h light-dark cycle), having free access to regular rodent chow and softened tap water. Sterile sunflower seeds and hydrogel were supplied as diet integration to prevent excessive body weight loss. All animal experiments described below were approved by the intramural animal-welfare committee for animal experimentation of Chiesi Farmaceutici under protocol number: 809/2020-PR and comply with the European Directive 2010/63 UE, Italian D.Lgs 26/2014 and with the Animal Research: Reporting of In Vivo Experiments (ARRIVE) guidelines [10]. A Visual Analogue scale (0-10) for pain assessment was assessed daily by a designated veterinarian or trained technicians. VAS $\geq 7$ and/or body weight loss $\geq 20 \%$ were considered as humane endpoints, as well as signs of dyspnoea or apathy evaluated by a designated veterinarian.

\section{Oropharyngeal administration}


The inhalation solution was composed of bleomycin (BLM) hydrochloride (Baxter Oncology GmbH, Germany) and Indocyanine Green (ICG, Sigma-Aldrich, St. Louis, MO) dissolved in $0.9 \% \mathrm{NaCl}$, at $1 \mathrm{mg} / \mathrm{mL}$ and $5 \mathrm{mg} / \mathrm{mL}$, respectively. BLM and ICG solutions (BLM+ICG) were mixed (volume ratio 1:1) and administered to 36 mice. ICG solution was diluted with saline $(2.5 \mathrm{mg} / \mathrm{mL})$ and administered to 15 mice enrolled as a control group (Sal+ICG).

Administration of either solution (day 0 ) was performed after mice were slightly anesthetized with $2.5 \%$ isoflurane (IsoFlo, Zoetis Inc., New Jersey, USA) delivered in a box. The animals were placed on an intubation platform, positioning their incisor on the wire, the tongue was then pulled out with forceps using a small laryngoscope and $50 \mu \mathrm{L}$ of solution was administered via oropharyngeal aspiration (OA) using a micropipette [11].

The operator kept the laryngoscope in place for 5-10 seconds to ascertain the correct and complete aspiration of the instilled liquid. Mice were held upright for 10-15 seconds before being placed back into the cage.

Three independent experiments were carried out. The whole experimental procedure is summarized in supplementary figure 1.

\section{Fluorescence imaging}

Animals were slightly anesthetized with $2 \%$ isoflurane, shaved and imaged using IVIS Lumina II (PerkinElmer Inc., Waltham, MA) [12].

ICG $\left(\lambda_{E x}=790 \mathrm{~nm}, \lambda_{\mathrm{Em}}=840 \mathrm{~nm}\right)$ fluorescent signal was quantified in calibrated (radiant efficiency) units ([photons $\left./ \mathrm{sec} / \mathrm{cm}^{2} / \mathrm{str}\right] /\left[\mu \mathrm{W} / \mathrm{cm}^{2}\right]$ ) in Sal+ICG and BLM+ICG mice using the software Living Image ${ }^{\circledR}$ version 4.3.1. (PerkinElmer Inc.).

Mice were imaged in prone position at 0, 7, 14 and 21 days after OA treatment; an average of total fluorescence signal emitted from the chest region was calculated for each mouse.

\section{Micro-Computed Tomography:}

Acquisition protocol and image post-processing analysis. Micro-Computed tomography (Micro-CT) was performed at day 7, 14 and 21 using a Quantum GX Micro-CT (PerkinElmer, Inc. Waltham, MA). Mice were slightly anesthetized with $2 \%$ isoflurane and images were acquired with the following parameters: $90 \mathrm{KV}$, $88 \mu \mathrm{A}$, total scan time of 4 minutes (over a total angle of $360^{\circ}$ ). The 'high speed' acquisition protocol was used, and a respiratory gated technique was applied. The entire set of projection radiographs was reconstructed using a filtered back-projection algorithm with a Ram-Lak filter and resulted in two stacks of 512 slices with a nominal resolution of $50 \mu \mathrm{m}$.

The reconstructed datasets were analysed with Analyze software (Analyze 12.0; Copyright 1986-2017, Biomedical Imaging Resource, Mayo Clinic, Rochester, MN). Following the analysis protocol, CT scans 
were filtered and then converted from grey levels to CT numbers (Hounsfield Units- HU).

For the quantitative assessment of the aeration degrees, the total lung volume was extracted from the reconstructed image $[11,13]$ through manual segmentation. the lung aeration compartments were then determined applying 'HU preclinical ranges' [13] dividing the whole parenchyma in normo-aerated ([-860, $-435] \mathrm{HU})$, hypo-aerated $([-435,-121] \mathrm{HU})$, non-aerated $([-121,+121] \mathrm{HU})$ and hyper-inflated areas $([-1040$, $-860] \mathrm{HU})$, expressed as percentage of total lung volume.

The hypo- and non-aerated lung tissues areas refer to those with a low gas/tissue ratio, which was previously developed to quantify lung fibrosis progression and evaluate antifibrotic drug efficacy [13].

\section{Micro CT Visual categorisation.}

Micro-CT scans were visually evaluated by three independent radiologists: four patterns were defined to categorize the lung parenchyma features (see supplementary figure 2). The final decisions reached by consensus.

Healthy lung was defined by homogenous density of lung parenchyma with regular-shaped blood vessels and airways. The reconstructed datasets revealed an entirely detectable lung volume, with normally aerated areas covering around $80 \%$ of total lung volume.

Typical BLM fibrosis (TBF) was defined by a patchy pattern with high attenuation areas randomly distributed within the lung parenchyma without visible low attenuation areas $[11,13,16]$.

Fibrotic lung associated with moderate airspace enlargement (FAE) was defined by a patchy pattern with heterogeneous density from hazy fibrotic pattern with moderate presence of low attenuation areas, which are difficult to isolate from the surrounding tissue.

Fibrotic lung with severe airspace enlargement $\left(\mathrm{FAE}^{+}\right)$was defined by a patchy pattern with heterogeneous density into an overt mosaic pattern characterized by multiple and markedly detectable low-density structures, with well-defined margins appearing in a bubble-like shape in lung parenchyma.

Each Micro-CT scan was attributed to one of these categories at days 7, 14 and 21 and the number of animals assigned to each category was calculated at every time point.

\section{Histological assessment of fibrosis}

At 7, 14 and 21 days, after the in-vivo imaging, subsets of 5 (Sal+ICG) and 12 (BLM+ICG) mice were euthanized with an overdose of anaesthetic followed by bleeding from the abdominal aorta. The lungs were removed and inflated with a cannula through the trachea by gentle infusion of $0.6 \mathrm{~mL}$ of $10 \%$ neutral-buffered formalin and fixed for $24 \mathrm{~h}$. For histological assessment, the samples were dehydrated in a graded ethanol series, clarified in xylene, and paraffin embedded. Sections of $5 \mu \mathrm{m}$ thickness were cut with a rotary microtome (Slee Cut 6062; Slee Medical, Mainz, Germany) and then stained with 
hematoxylin and eosin (H\&E) and Masson's trichrome (TM), according to the manufacturer's specifications (Histo-Line Laboratories). The whole slide images (WSI) were acquired by the NanoZoomer S-60 Digital slide scanner (Hamamatsu, Japan) for analysis. Three sections for each lung sample were stained with TM and scored on a scale of 0 to 8 by two independent investigators who were blinded to the treatments. Fibrotic modifications were assessed morphologically and semi-quantitatively graded according to the scale defined by Ashcroft et al. [14] and modified by Hübner et al [15]. The final score was expressed as a mean of individual scores observed across all microscopic fields. To quantify the distribution of pulmonary fibrosis, the Ashcroft scores were graded in three classes of increasing values ranging from 0 to 3 (mild), 4 (moderate), and $\geq 5$ (severe).

\section{Histo-morphometric assessment of airspace enlargements}

Alveolar airspace enlargement and alveolar destruction, in accordance with emphysema definition [17], were histomorphometrically evaluated by the parameters of Mean Linear Intercept (MLI) and alveolar airspace area (AAA) [18-23]. The alveolar air spaces area was dimensionally categorized for the analysis as normal $(0-100 \mu \mathrm{m})$, medium $(101-300 \mu \mathrm{m})$, large $(\geq 300 \mu \mathrm{m})$, labeled in white, grey and black, respectively. The distribution of AAA during the time course was expressed as percentage of air content normalized to the total lung parenchyma. See supplementary methods for more details.

\section{Statistics}

Statistical analyses were performed using Prism 8 software (GraphPad Software Inc., San Diego, CA, United States). All data are presented as mean \pm SEM. Two-way analysis of variance (ANOVA) was performed, followed by Sidak's multiple comparison post hoc test. Normal distribution was assessed through Shapiro-Wilk test accompanied by visual inspection of QQ-plots. Sample size was calculated with A-priori Power Analysis (G*Power Version 3.1.2) considering Ashcroft Score as endpoint. For all the applied tests, a $p$-value $<0.05\left(^{*}\right)$ was considered as statistically significant.

\section{Results}

\section{Fluorescence imaging and prolonged ICG retention in lungs}

In-vivo fluorescence of Sal+ICG and BLM+ICG mice in prone position and ex-vivo lung signals at different time points are shown in Figure $1 \mathrm{~A}$ and $\mathrm{B}$. The images showed a fluorescent signal well localized in the chest region in all mice. The quantification of fluorescent signal did not show any statistically significant difference between Sal+ICG and BLM+ICG treated mice at any time (Figure 1C and D).

The signal was present at the time of $\mathrm{OA}$ and through the pre-defined time points, in particular: fluorescence peaked at day 7 and gradually decreased at later time points, either in-vivo or ex-vivo. At day 21, a conspicuous ICG signal was still detected (7 and 9 Log radiant efficiency, respectively) (Figure 1C, D). 
These results clearly indicate a remarkable persistence of ICG in the lungs when the dye is administered alone or in association with bleomycin. The in-vivo imaging consolidated two hypotheses: first, ICG can be used as a tracer to map the distribution and exposure of BLM in lungs, and second, OA is confirmed as a suitable route of administration to obtain uniform lung distribution of the instilled solution [11].

\section{Micro-CT analysis}

The representative Micro-CT imaging of 3D lung segmentation at different time points (Figure 2A) showed hypo- and non-aerated areas, associated with appearance of hyper-inflated lung tissue in $B L M+I C G$ mice, whilst Sal+ICG treated animals had normal lung parenchyma throughout the time course.

Hypo- and non-aerated tissues were used to classify the lung compartments with different gas/tissue ratio corresponding to moderate or severe fibrosis.

The time course quantification of lung aeration degrees (Figure 2B) showed a significant increase in hypo- and non-aerated tissues already at day 7 with peak for both compartments at day 14 .

At day 21 , a significant increase $(p<0.05)$ of normo- and a reduction of hypo-aerated tissues were observed compared to 14 day, whilst no significant variations occurred regarding non-aerated areas. Notably, a significant amount of hyper-inflated tissue was detected along the time course of the experiment, highest at day 21 .

\section{Micro-CT scans visual categorization}

Sal+ICG treated mice showed a healthy lung parenchyma at each time point of observation.

The three morphologic categories were seen in BLM+ICG mice (Figure 4A), in particular: at day 7, TBF pattern was observed in 5 out of 12 of subjects $(42 \%)$ while the remaining $7(58 \%)$ were FAE (Figure 4B, supplementary table 2$)$. As regards day 14 , FAE was still the prevailing pattern ( 7 of $12,58 \%$, whereas TBF was reduced to 3 of $12(25 \%)$, and FAE+ appeared in 2 of $12(17 \%)$ of the subjects. At day $21,6 \mathrm{FAE}^{+}$ patterns (50\%), 2 TBF (17\%) and 4 FAE (33\%) were recorded.

$\mathrm{BLM}+\mathrm{ICG}$ mice were classified as TBF, FAE and $\mathrm{FAE}^{+}$and lung aeration degrees were quantified (Figure 4C), to compare different categories regardless of the time-point.

The FAE, with respect to TBF class, showed a markedly higher $(\mathrm{p}<0.01)$ hypo- and non-aerated tissue, as well as reduced normo-aerated areas, whilst the hyper-inflated tissue was augmented although not statistically significantly. $\mathrm{FAE}^{+}$category, as compared with TBF, exhibited a significantly lower normoaerated tissue $(\mathrm{p}<0.01)$, whereas hypo- and non-aerated areas were not significantly different, although slightly higher than TBF. Finally, a significant increase in hyper-inflated tissue $(p<0.05)$ was observed in $\mathrm{FAE}^{+}$with respect to TBF.

\section{Histological assessment of lung fibrosis}


Histological analysis was performed on subsets of Sal+ICG and BLM+ICG mice sacrificed at 7, 14 or 21 days. BLM+ICG histology exhibited a patchy pattern of severe lung fibrosis, characterized by an increasing deposition and compactness of the substitutive extracellular matrix within the fibrotic foci. This pattern was already well-established at day 7 , worsened at day 14 and remained stable up until day 21 (Figure 3A). Moreover, the destruction and collapse of alveolar walls with alveolar airspaces enlargement, already observed at day 7 , tended to evolve at 14 and 21 days, along with widening of the balloon-like airspace resembling emphysema-like morphology. (Figure 3A). The Sal+ICG group instead, showed normal lung architecture throughout the study (Figure 3A). Ashcroft score revealed a severe lung fibrosis and was significantly increased $(p<0.001)$ at all time points, although slightly higher at day 14 , in BLM+ICG compared to Sal+ICG treated mice (Figure 3B). Ashcroft score Frequency distribution grouped as mild $(0-3)$, moderate $(4)$, and severe $(\geq 5)$ significantly changed $(p<0.05)$ as compared to Sal+ICG, revealing a prevalence of severe fibrosis at each time point, with a peak at 14 days, while the moderate values remained stable for BLM+ICG mice during time (Figure $3 \mathrm{C}$ ).

\section{Histo-morphometric evaluation of airspace enlargements.}

Representative micrographic images of Sal+ICG and BLM+ICG groups at different time are shown in figure 5 .

The BLM+ICG group showed significantly more large airspaces and less medium airspaces at day 14 $(p<0.05)$, compared to Sal+ICG group. This pattern of airspace abnormalities was even more pronounced at day $21(p<0.05)$.

These data were confirmed by MLI, which showed significant structural changes at day 21 in the BLM+ICG group (Figure 5C).

Changes in AAA and MLI described disease progression resembling an emphysema-like pattern, in agreement with the outcomes arising from Micro-CT analysis, which were characterized by multiple and markedly detectable low-density structures, appearing in a bubble-like shape in the lung parenchyma.

\section{Discussion}

One of the novelties provided by this study is the introduction of a simple in-vivo method to follow lung distribution of BLM. This approach can help reduce variability within and between experimental groups in preclinical studies on lung fibrosis, leading to the reduction of sample size, thus improving compliance with the $3 R^{\prime}$ s principles.

This study showed that the mouse model with OA of BLM + ICG grants homogeneous distribution of compounds to lung parenchyma, as shown by in-vivo and ex-vivo fluorescence signals. The solution of BLM and ICG reflected one of a kind pulmonary damage with long-term evolution of hypoattenuating lung areas on micro-CT and histological evidence of severely enlarged airspaces in association with severe fibrosis, different from TBF. 
IPF represents an urgent unmet medical need. Thus, it is imperative to develop disease-modifying therapies, for which purpose refined animal models are crucial. The first objective of the present study was to set up a method to monitor the distribution of BLM after OA through fluorescence imaging. Indeed, although performing $O A$ requires lower technical skills with respect to intratracheal instillation, variability in terms of lung exposure remains a common issue, leading to biased outcomes and increasing the number of animals required to produce statistically significant results.

Administration via OA generated homogeneous lung distribution as revealed by the quantification of invivo and ex-vivo fluorescence signals (Fig. 1), confirming that ICG can be used as a tracer to early identify mice that for technical reasons would exhibit a lower or non-uniform lung exposure.

However, Micro-CT and histological analyses revealed a more severe lung fibrosis compared to our classical BLM model, even though a single rather than a double administration via OA was applied [11, $13,16]$.

A severe lung fibrosis appeared as soon as day 7 , peaked at 14 days and remained stable up to day 21 . In fact, a manual segmentation of micro-CT images was necessary for the BLM + ICG mice to detect nonaerated areas (Fig. 2A, B), in line with the Ashcroft frequency distribution (Fig. 3B) revealing the prevalence of severe lesions at every time point. The coupling of the destruction and collapse of the alveolar walls with alveolar airspaces enlargement evolved in a severe fibrotic lesion interconnected with emphysema-like morphology, characterized by balloon-like appearance of parenchyma and producing a resemblance to honeycombing (Fig. 3A).

Other distinctive features highlighted by Micro-CT, compared to typical BLM fibrosis, were low density areas with a bubbles-like shape of hyper-inflated tissue (Fig. 4A, supplementary Fig. 2) which appeared at days 7 and 14 and increased at day 21. These lung tissue structures, resembling emphysema-like features, were categorized as TBF, FAE and $\mathrm{FAE}^{+}$through a visual Micro-CT scans classification at the different time-points (Fig. 4B). These features have not been reported in our previous studies on BLMinduced fibrosis $[11,16]$, representing distinctive hallmarks of a BLM + ICG induced lung disease.

The categories identified by visual score were also significantly different in terms of lung aeration degrees (Fig. 4C), as mice classified as $\mathrm{FAE}^{+}$showed a 3-fold increase in hyper-inflated areas compared to other categories.

This evolution was consistent with substantial prevalence of long-term $\mathrm{FAE}^{+}$, under the newly developed BLM + ICG condition. In line with these findings, histo-morphometric evaluation of airspace size revealed severe abnormalities at day 21 (Fig. 5A), as confirmed by the significant increase in AAA and MLI (Fig. 5B, C).

The progressive lung disease with severe fibrosis and emphysema-like features reported by both MicroCT and histo-morphometric analyses cannot be attributed to the tracer itself, as animals receiving saline + ICG had healthy lungs. ICG is considered a safe and biologically inactive probe, as in over 40 years of 
clinical practice adverse reactions have been anecdotal [24]. Nonetheless, in our study the combination of BLM and ICG triggered a disease that was clearly different from the typical BLM-induced lung fibrosis with a single administration corresponding to half of the dose we routinely use [16.] ICG is known to have a very short half-life and to be rapidly eliminated by the liver [25] upon intravenous administration, which seems to be in contrast with our findings, as we detected a remarkable fluorescence signal in lungs up to 21 days (Fig. 1). However, there is robust evidence indicating that ICG strongly binds to proteins and phospholipids (PL), which deeply affect the fluorescence yield and half-life [26, 27] of the tracer. For this reason, in clinical practice, different ICG-based formulas in which the dye is combined with proteins have been used to improve the retention in target organs [28, 29]. Lung surfactant being composed mainly of PL and proteins ${ }^{30}$ we could hypothesize that interaction between ICG and surfactant's components led to the prolonged retention of the probe within the lungs, despite surfactant deprivation being postulated to occur in models of lung fibrosis [31].

We could hypothesize that the prolonged retention of ICG in the lungs exacerbated the pulmonary damage triggered by bleomycin. However, a thorough mechanistic explanation has yet to be clarified and represents one of the key steps for future studies.

\section{Conclusions}

In the present work, we have described a model of pulmonary fibrosis with peculiar characteristics that may be added to the list of experimental tools to be exploited in drug discovery, to foster the development of more effective therapeutics.

In addition, a common issue in pharmacological studies based on the BLM model is that fibrosis starts to spontaneously resolve after 14 days, thus hindering the achievement of a proper time window to reveal the efficacy of potential new drugs. Notably, in this model the fibrosis develops early and does not seem to be resolving at 21 days, as well as being associated with progressive emphysema -like features, resembling human IPF $[32,33]$.

Although, significant anatomical alterations were reported, namely emphysema, further investigations will be required to elucidate the mechanisms underlying the development of the disease.

We suppose that airspace size and hyper-inflated areas increase could be driven by a severe fibrosis exerting traction on lung parenchyma, together with lung functional alteration.

Functional measurements, such as functional Micro-CT analysis, could provide a more extensive comprehension of the disease progression.

However, further investigations will be required to elucidate the mechanisms underlying the development of fibrosis with enlarged airspaces elicited by BLM + ICG. 
To sum up, we have highlighted the pivotal role of Micro-CT technology in animal models of lung disease, unveiling the hallmarks of disease progression by both visual and quantitative methods. We strongly believe that a continuous refinement of animal models together with an integrative approach with MicroCT and other imaging technologies represents a potential complement for clinical translatability.

\section{Abbreviations}

BLM: Bleomycin

ICG: Indocyanine Green

IPF: Idiopathic Pulmonary Fibrosis

CT: $\quad$ Computed Tomography

TBF: Typical bleomycin fibrosis

FAE: Fibrotic with moderate airspaces enlargements

$\mathrm{FAE}^{+}$: Fibrotic with severe airspaces enlargements

MLI: Mean Linear Intercept

AAA: Alveolar Airspace Area

OA: Oropharyngeal Aspiration

HU: Hounsfield Units

H\&E: Hematoxylin Eosin

TM: Masson's Trichrome

VAS: Visual Analogue Scale

PL: Phospholipids

Sal: Saline

IVIS: In-vivo Imaging System

\section{Declarations}

Availability of data and materials. 
The datasets used and/or analysed during the current study are available from the corresponding author on reasonable request.

\section{Acknowledgments.}

The authors wish to thank Mark Watling for language editing.

\section{Funding.}

This study was fully supported by Chiesi Farmaceutici S.p.A

\section{Competing interests' statement.}

FS, GV and AG are employees of Chiesi Farmaceutici S.p.A., that supported the research work.

The remaining authors declare that the research was conducted in the absence of any commercial or financial relationships that could be construed as a potential conflict of interest.

\section{Authors' contributions}

$A G, E F$ and $F F S$ conceived and designed research. $A G, E F, R C$ and $F F S$ performed experiments. $M M, L L$, $L M, A G$ analysed data. AG, MS, NS, FFS, LR interpreted results. AG, EF, RC, MM prepared figures. AG, FFS drafted manuscript. GV, GD, MS, LR, FFS edited and revised manuscript. All authors approved final version of manuscript.

\section{Ethics statement.}

All animal experiments described below were approved by the intramural animal-welfare committee for animal experimentation of Chiesi Farmaceutici under protocol number: 809/2020-PR and comply with the European Directive 2010/63 UE, Italian D.Lgs 26/2014.

\section{References}

1. Martinez FJ, Collard HR, Pardo A, et al. Idiopathic pulmonary fibrosis. Nature Reviews Disease Primers. 2017;3:17074.

2. Kumar A, Kapnadak SG, Girgis RE, Raghu G. Lung transplantation in idiopathic pulmonary fibrosis. Expert Review of Respiratory Medicine. 2018;12:375-85.

3. Chua F, Gauldie J, Laurent GJ. Pulmonary fibrosis: searching for model answers. Am J Respir Cell Mol Biol. 2005;33:9-13.

4. Moore BB, Lawson WE, Oury TD, et al. Animal models of fibrotic lung disease. Am J Respir Cell Mol Biol. 2013;49:167-79.

5. Jenkins RG, Moore BB, Chambers RC, et al. An official American thoracic society workshop report: Use of animal models for the preclinical assessment of potential therapies for pulmonary fibrosis. 
Am J Respir Cell Mol Biol. 2017;56:667-79.

6. Henderson NC, Rieder F, Wynn TA. Fibrosis: from mechanisms to medicines. Nature. 2020;587:55566.

7. Mizuno T, Mohri K, Nasu S, et al. Dual imaging of pulmonary delivery and gene expression of dry powder inhalant by fluorescence and bioluminescence. J Controll release. 2009;134(2):139-54.

8. Chiu $\mathrm{CH}$, Chao YK, Liu YH, et al. Clinical use of near-infrared fluorescence imaging with indocyanine green in thoracic surgery: a literature review. J Thorac Dis. 2016;8(Suppl 9):744-8.

9. Liu Y, Peng J, Wang S, et al. Molybdenum disulfide/graphene oxide nanocomposites show favorable lung targeting and enhanced drug loading/tumor-killing efficacy with improved biocompatibility. NPG Asia Mater. 2018;10:e458.

10. du Sert NP, et al. Reporting animal research: Explanation and elaboration for the arrive guidelines 2.0. PLoS Biol. 2020;18:e3000411.

11. Ruscitti F, Ravanetti F, Donofrio G, et al. A Multimodal Imaging Approach Based on Micro-CT and Fluorescence Molecular Tomography for Longitudinal Assessment of Bleomycin-Induced Lung Fibrosis in Mice. Journal of Visualized Experiments. 2018.

12. Stellari F, Sala A, Ruscitti F, et al. Monitoring inflammation and airway remodeling by fluorescence molecular tomography in a chronic asthma model. J Transl Med. 2015 Oct;24:13:336.

13. Mecozzi L, Mambrini M, Ruscitti F, et al. In-vivo lung fibrosis staging in a bleomycin-mouse model: a new micro-CT guided densitometric approach. Sci Rep. 2020 Oct;30(1):18735. 10(.

14. Ashcroft T, Simpson JM, Timbrell V. Simple method of estimating severity of pulmonary fibrosis on a numerical scale. J Clin Pathol. 1988;41:467-70.

15. Hübner RH, Gitter W, El Mokhtari NE, et al. Standardized quantification of pulmonary fibrosis in histological samples. Biotechniques. 2008;44(4):507-17.

16. Ferrini E, Mecozzi L, Corsi L, et al. Alfaxalone and Dexmedetomidine as an Alternative to Gas Anesthesia for Micro-CT Lung Imaging in a Bleomycin-Induced Pulmonary Fibrosis Murine Model. Front Vet Sci. 2020 Oct 8;7:588592.

17. Kinoshita $\mathrm{Y}$, Watanabe $\mathrm{K}$, Ishii $\mathrm{H}$, et al. Distribution of emphysema and fibrosis in idiopathic pulmonary fibrosis with coexisting emphysema. Histopathology. 2019 Jun;74(7):1103-8.

18. Bai J, Qiu SL, Zhong XN, Huang QP, He ZY, Zhang JQ, et al. Erythromycin enhances CD4 + Foxp3 + regulatory T-cell responses in a rat model of smoke-induced lung inflammation. Mediat Inflamm. 2012;2012:410232.

19. Duan MC, Huang Y, Zhong XN, Tang HJ. Th17 cell enhances CD8 T-cell cytotoxicity via IL-21 production in emphysema mice. Mediat Inflamm. 2012;2012:898053.

20. Eidelman DH, Ghezzo H, Kim WD, Cosio MG. The destructive index and early lung destruction in smokers. Am Rev Respir Dis. 1991;144:156.

21. Goldstein I, Bughalo M, Marquette C, Lenaour G, Lu Q, Rouby J. Mechanical ventilation-induced airspace enlargement during experimental pneumonia in piglets. Am J Respir Crit Care Med. 
2001;163:958.

22. Zhang H, Zhou X, Chen X, Lin Y, Qiu S, Zhao Y, Tang Q, Liang Y, Zhong X. Rapamycin attenuates Tc1 and Tc17 cell responses in cigarette smoke-induced emphysema in mice. Inflamm Res. 2019 Nov;68(11):957-68.

23. Crowley G, Kwon S, Caraher EJ, et al. Quantitative lung morphology: semi-automated measurement of mean linear intercept. BMC Pulm Med. 2019 Nov;9(1):206. 19(.

24. Hope-Ross M, Yannuzzi LA, Gragoudas ES, Guyer DR, Slakter JS, Sorenson JA, Krupsky S, Orlock DA, Puliafito CA. Adverse reactions due to indocyanine green. Ophthalmology. 1994 Mar;101(3):529-33.

25. Carelli S, Giallongo T, Rey F, et al. Neural precursors cells expanded in a 3D micro- engineered niche present enhanced therapeutic efficacy in vivo. 2021; 5(1): 8-26.

26. Desmettre T, Devoisselle JM, Mordon S. Fluorescence properties and metabolic features of indocyanine green (ICG) as related to angiography. Surv Ophthalmol. 2000 Jul-Aug;45(1):15-27.

27. Miranda D, Wan C, Kilian HI, Mabrouk MT, Zhou Y, Jin H, Lovell JF. Indocyanine green binds to DOTAP liposomes for enhanced optical properties and tumor photoablation. Biomater Sci. 2019 Aug $1 ; 7(8): 3158-3164$.

28. Verbeek FP, Tummers QR, Rietbergen DD, et al. Sentinel Lymph Node Biopsy in Vulvar Cancer Using Combined Radioactive and Fluorescence Guidance. Int J Gynecol Cancer. 2015;25(6):1086-93.

29. Hutteman M, van der Vorst JR, Gaarenstroom KN, et al. Optimization of near-infrared fluorescent sentinel lymph node mapping for vulvar cancer. Am J Obstet Gynecol. 2012;206(1):89.e1-5.

30. Bernhard W. Lung surfactant: Function and composition in the context of development and respiratory physiology. Ann Anat. 2016 Nov;208:146-50. doi:10.1016/j.aanat.2016.08.003. Epub 2016 Sep 29.

31. Lopez-Rodriguez E, Boden C, Echaide M, et al. Surfactant dysfunction during overexpression of TGF1 precedes profibrotic lung remodeling in vivo. Am J Physiol Lung Cell Mol Physiol. 2016;310:L1260 $-1271$.

32. Cottin V. Combined pulmonary fibrosis and emphysema: bad and ugly all the same? Eur Respir J. 2017 Jul 5;50(1):1700846.

33. Pepin EW, Verma N, Mehta HJ, Mohammed TL. Combined Pulmonary Fibrosis and Emphysema. Ann Am Thorac Soc. 2018 Jan;15(1):110-2.

\section{Figures}




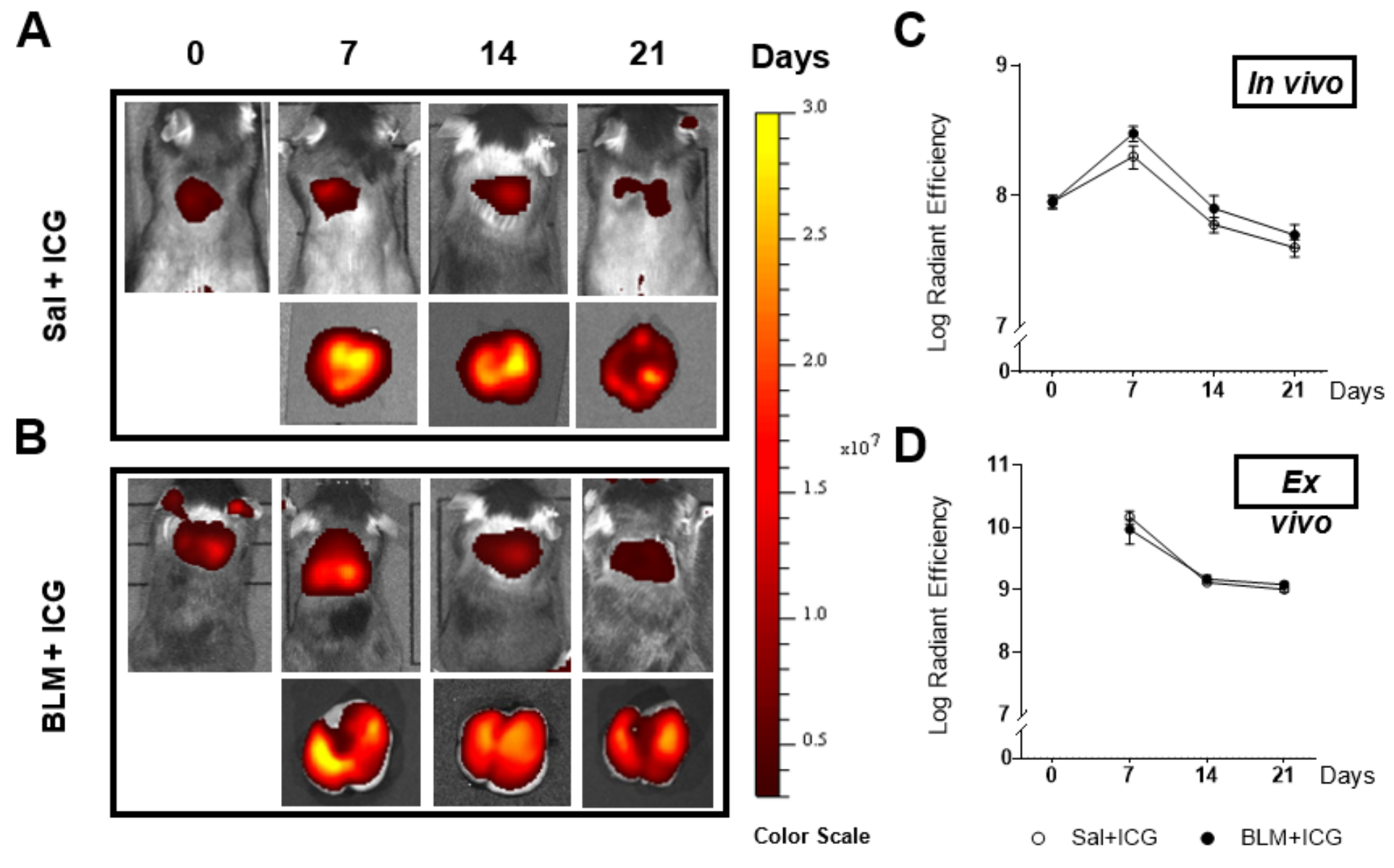

Figure 1

In-vivo and ex-vivo fluorescence imaging. Representative images of Sal+ICG and BLM+ICG mice at different time-points $(0,7,14$, and 21 days after $O A)(A, B)$. Time course of the quantified signals of invivo (C) and ex-vivo (D) fluorescence. Data plotted as mean $\pm \mathrm{sem}$. Numbers of animals were as follows: Sal+ICG 5 animals at each time point, BLM+ICG 12 animals at each time point. 3 independent experiments were carried out. 


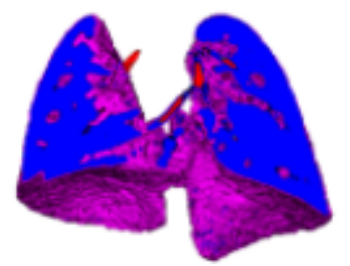

Sal+ICG

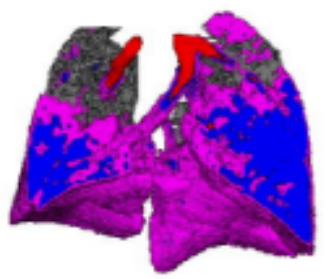

7

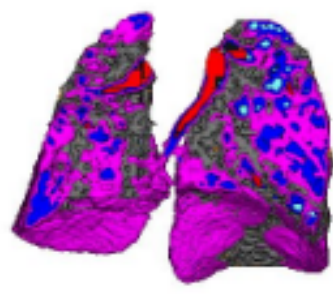

14

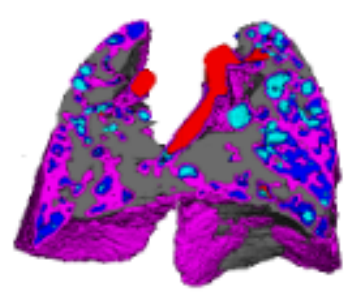

21

\section{$B L M+I C G$}

B

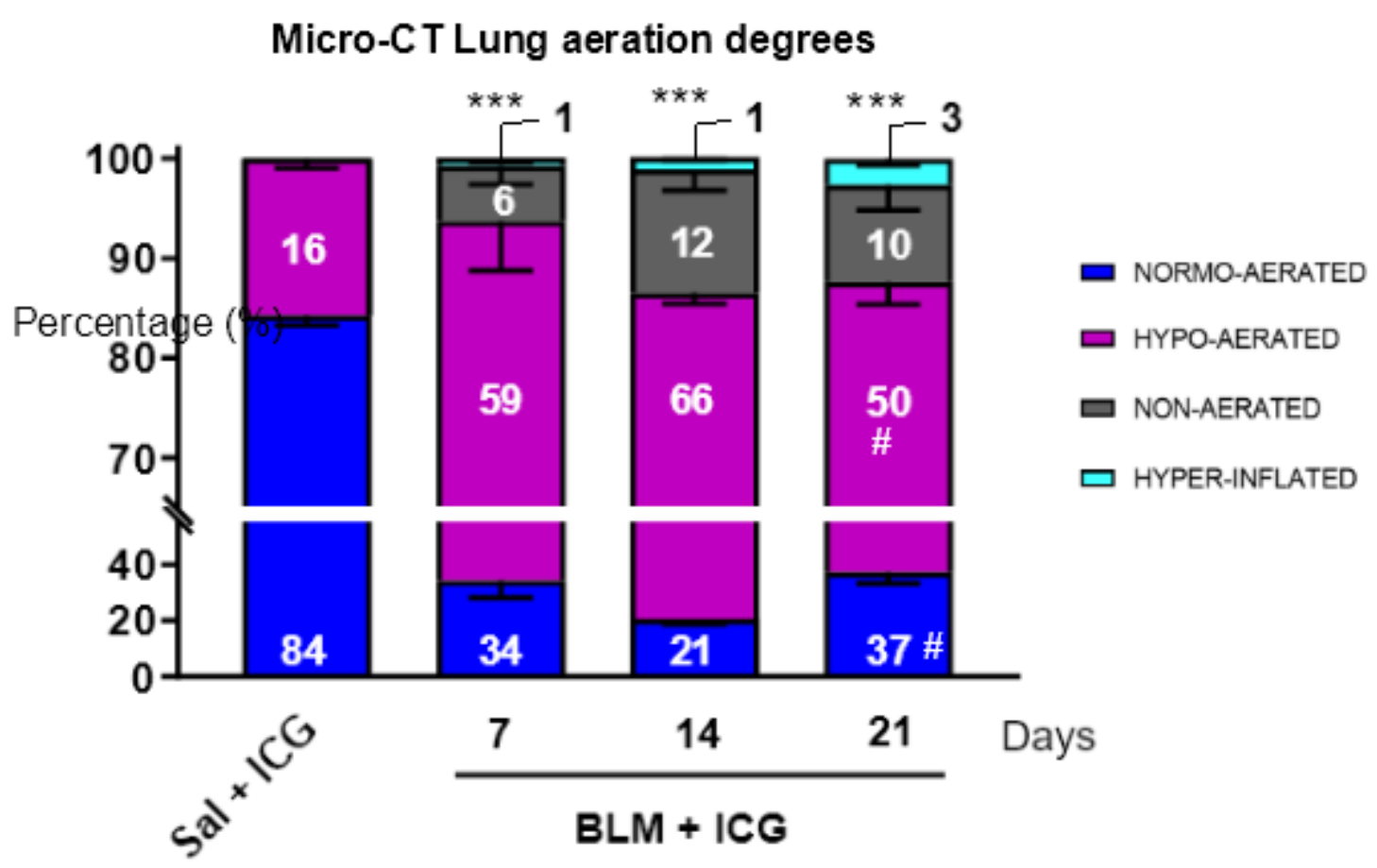

Figure 2

Quantitative assessment of lung aeration degrees by Micro-CT. representative 3D lung renderings of Sal+ICG and BLM+ICG mice at 7, 14 and 21 days (A): In the Sal+ICG group no alterations in the parenchyma occurred between the time-points. Airways are in red. Percentage of normo-, hypo-, nonaerated and hyper-inflated tissues revealed by Micro-CT in Sal+ICG and BLM+ICG mice at 7, 14 and 21 days (B). Values shown by the Sal+ICG group were constant throughout all time-points. Two-way ANOVA with Sidak's correction for multiple comparison was applied on the following numbers of animals: Sal + ICG $n=5$ at each time point; BLM+ICG $n=12$ at each time point. ${ }^{* \star *} p<0.001$ vs Sal+ICG at respective timepoint for all compartments. \# $p<0.05$ vs previous time-point. Data were collected from 3 independent experiments. 


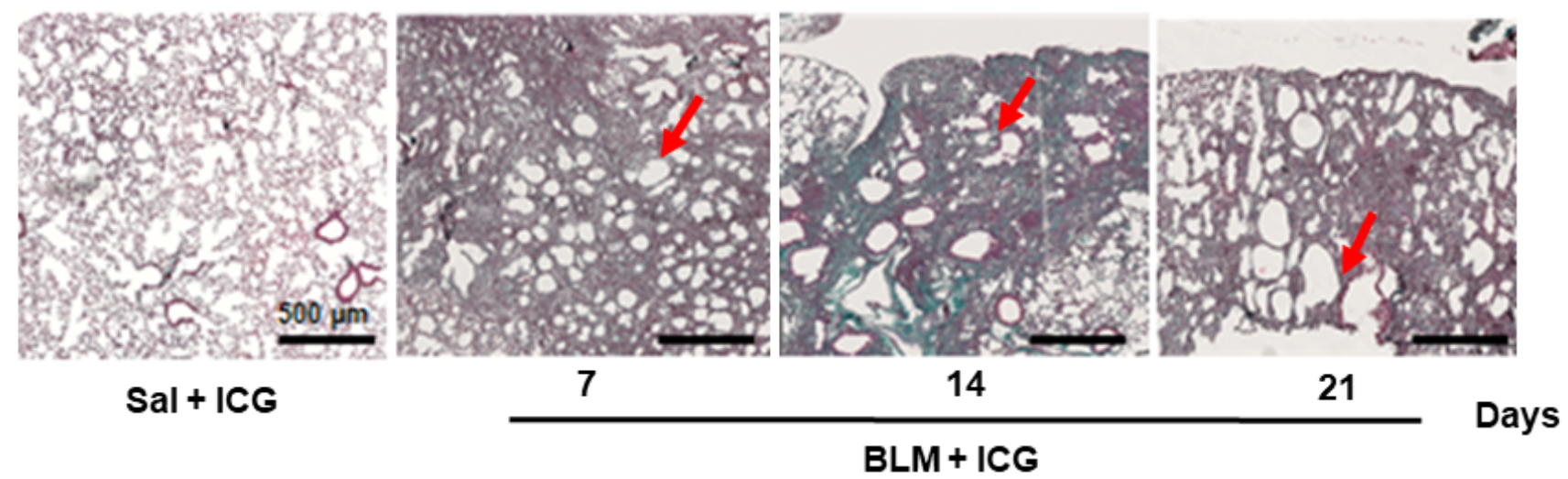

B

\section{Ashcroft Score}

\section{C}
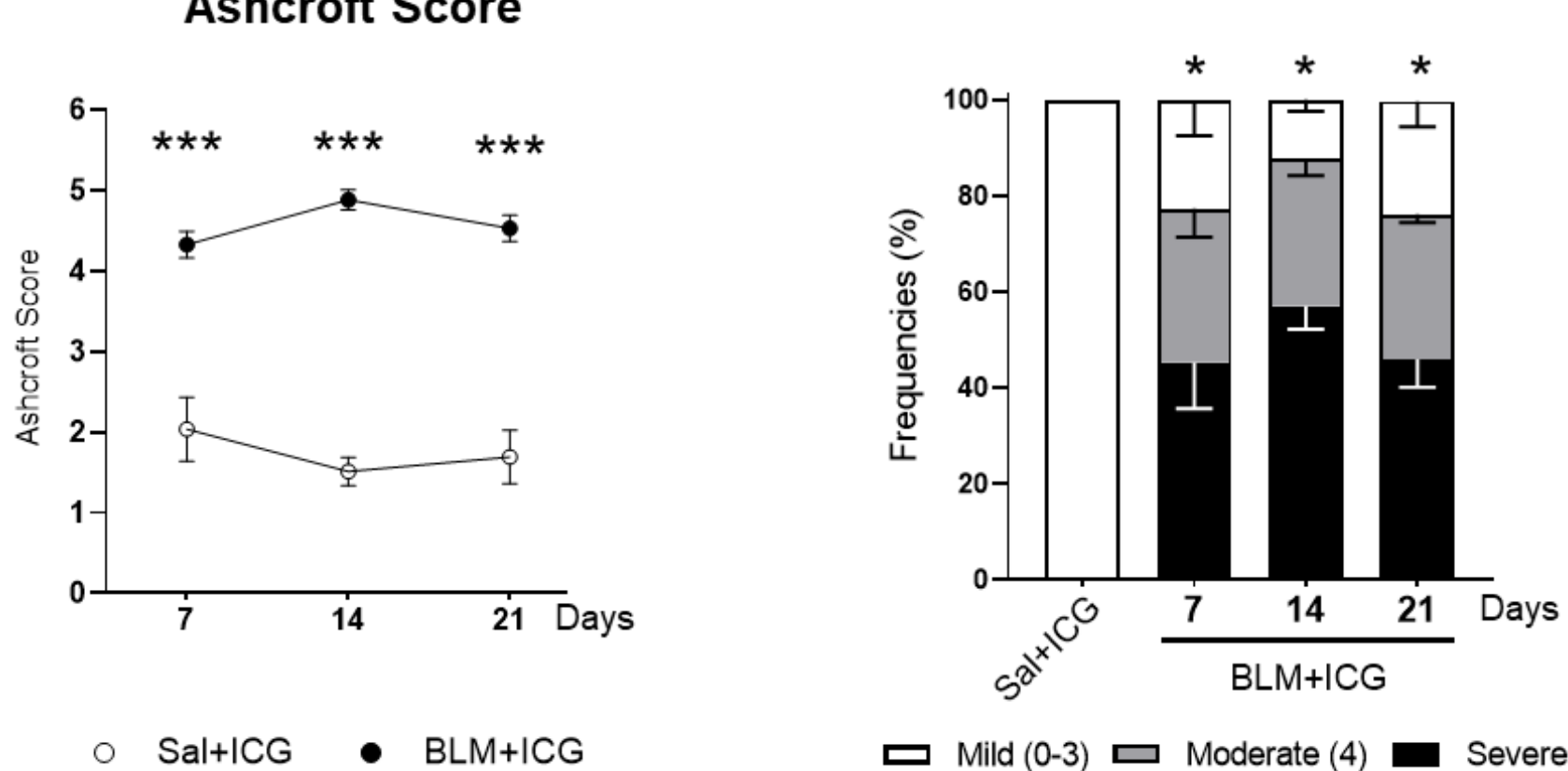

$\square$ Mild (0-3) $\square$ Moderate (4) $\square$ Severe ( $\geq 5)$

\section{Figure 3}

Histological assessment of lung fibrosis. Representative whole slide images of Masson's trichrome stained section of lungs of Sal+ICG and BLM+ICG mice at days 7, 14 and 21 (5X magnification; scale bars $500 \mu \mathrm{m})(\mathrm{A})$. Time course of the Ashcroft Score (B) and its frequency distribution (C) determined at days 7, 14 and 21 on Sal+ICG and BLM+ICG mice. Frequency distribution in Sal+ICG mice remained constant throughout all endpoints. Two-way ANOVA with Sidak's correction for multiple comparison was applied on the following number of animals: Sal+ICG $n=5$ at each time point; $B L M+I C G n=12$ at each time point. ${ }^{\star} p<0.05 ;{ }^{* \star} p<0.01 ;{ }^{* \star \star} p$-value $<0.001$ vs Sal+ICG at respective time-point. Data collected from 3 independent experiments. 
A

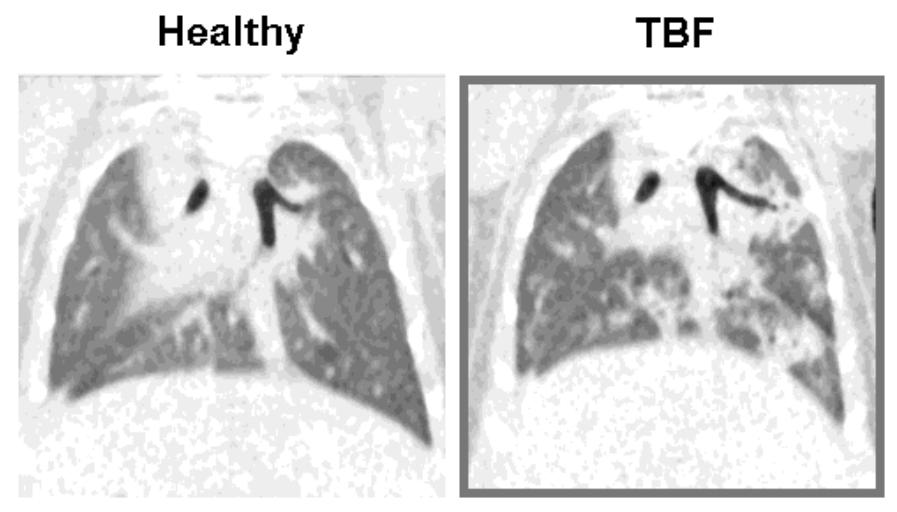

FAE

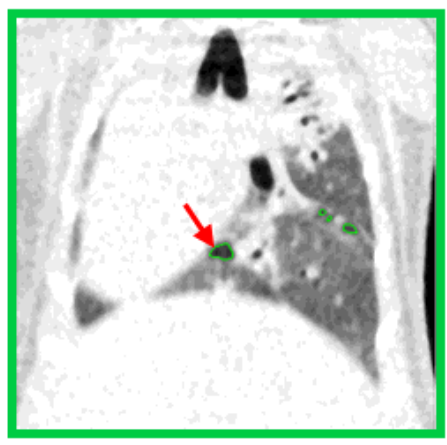

C
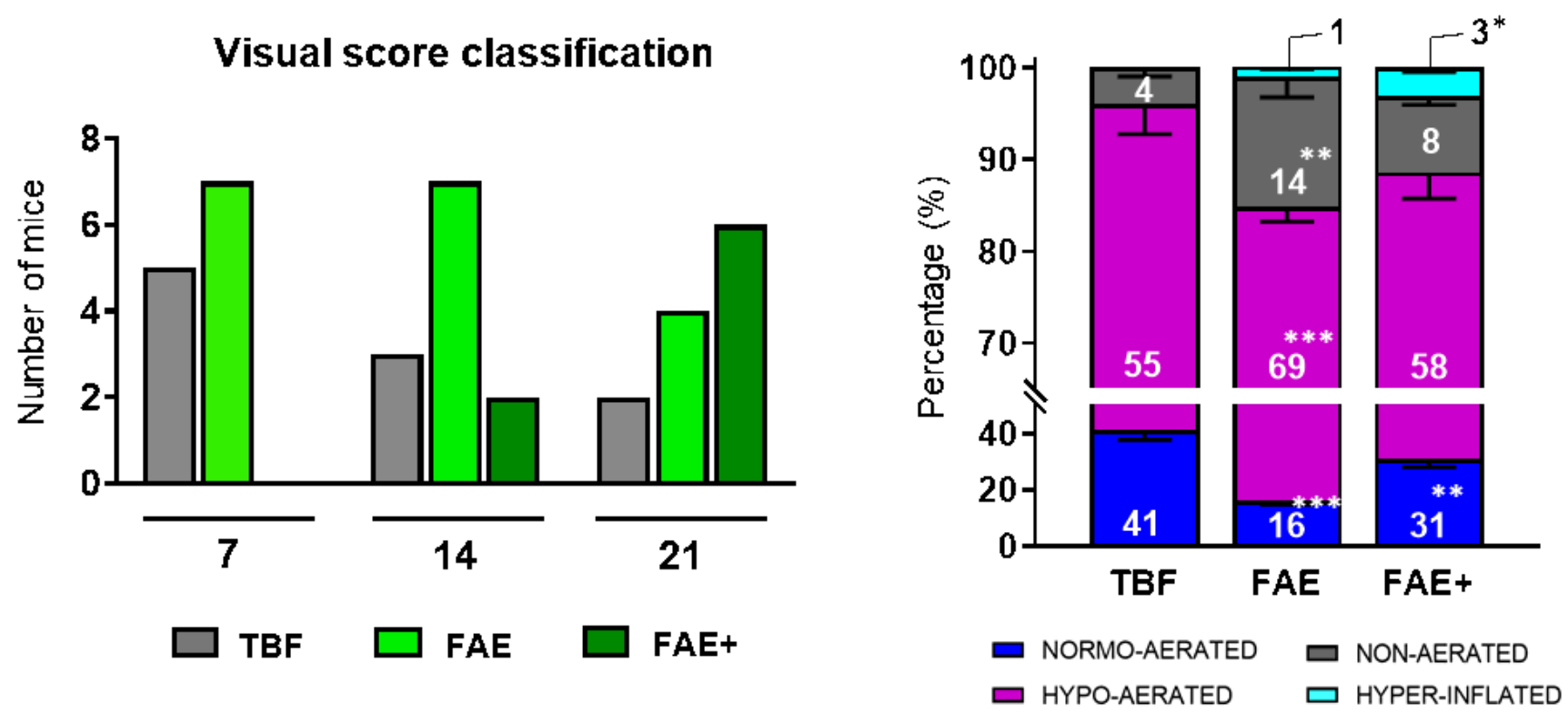

Figure 4

Visual categorization of Micro-CT scans. Four different patterns of lung parenchyma were identified by Micro-CT. Representative images of each pattern in (A). Red arrows and green dots indicate low attenuation areas. Visual score classification for each BLM+ICG animal at days 7, 14 and 21 (B). Percentage of normo-, hypo-, non-aerated and hyper-inflated tissues revealed by Micro-CT in BLM+ICG mice classified as TBF, FAE and FAE $+(C)$. Data expressed as mean \pm sem. ${ }^{\star} p<0.05 ;{ }^{* \star} p<0.01$; ${ }^{* \star *} p-$ value $<0.001$ vs TBF. Data were collected from three independent experiments. 
A $\operatorname{large}(\geq 300 \mu \mathrm{m}) \square$ medium $(100-300 \mu \mathrm{m}) \quad \square$ normal $(0-100 \mu \mathrm{m})$

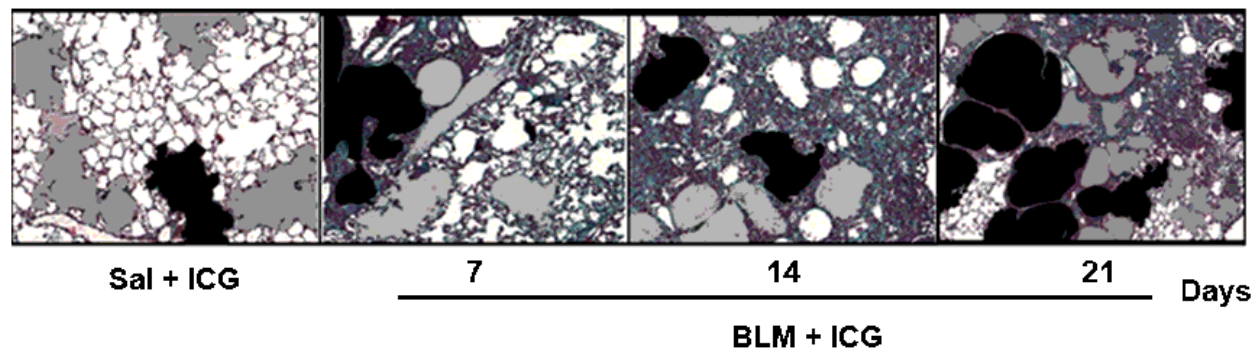

B

\section{Alveolar Air Spaces}

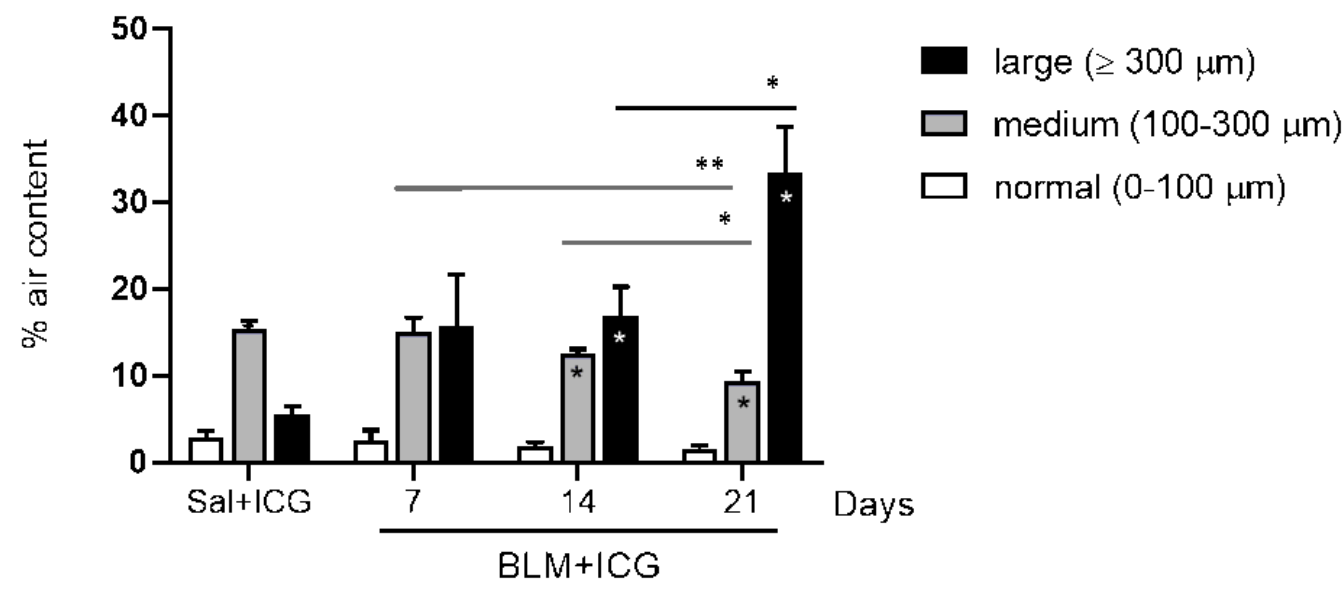

C

Mean Linear Intercept (MLI)

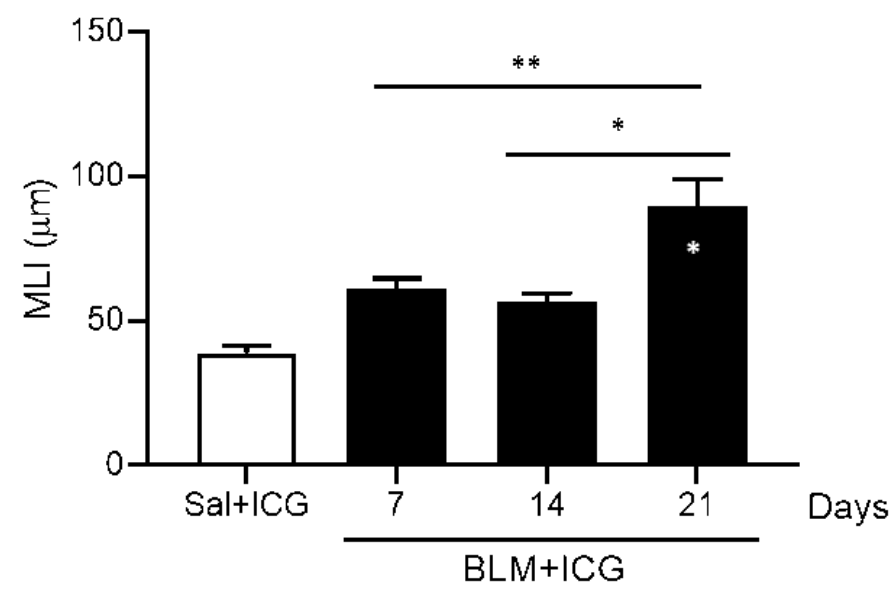

\section{Figure 5}

Histo-morphometric evaluation of airspace enlargements. Representative images of alveolar airspaces categories in mice lungs, analysed with Visiopharm ${ }^{\circledR}$ system and showed with original histological colours, Masson's Thricrome stain. The different airways size showed in Sal+ICG and BLM+ICG at days 7, 14 and 21, are displayed: normal airways category (0-100 um), medium airways category (101-300 um) large airways category ( $\geq 300 \mathrm{um}$ ) (A). Percentage of air content in the alveolar air spaces categories; 
asterisks within the bars indicate significant differences from saline, while asterisks above the horizontal lines indicate significant differences between 7-14-21 days ( $\left.{ }^{* *} p<0.01 ;{ }^{*} p<0.05\right)$ (B). Alveolar air spaces evaluated by mean linear intercept $(M L I)$, asterisks within the bars indicate significant differences from saline, while asterisks above the horizontal lines indicate significant differences between 7-14-21 days $\left({ }^{\star *} p<0.01 ;{ }^{*} p<0.05\right)(C)$.

\section{Supplementary Files}

This is a list of supplementary files associated with this preprint. Click to download.

- supplementarymaterials.docx

- Supplementaryfigures.docx 